\title{
Prevalencia de sintomáticos respiratorios, de infección y enfermedad tuberculosa y factores asociados: estudio basado en población, Mitú, Vaupés, 2001
}

\author{
Ingrid García ${ }^{1}$, Fernando De la Hoz 1,2, Yolima Reyes ${ }^{3}$, Pablo Montoya ${ }^{3}$, \\ Martha Inírida Guerrero ${ }^{4}$, Clara Inés León ${ }^{4}$ \\ 1 Subdirección de Epidemiología y LNR, Instituto Nacional de Salud, Bogotá, D.C., Colombia. \\ 2 Programa Nacional de Ciencia y Tecnología de la Salud, Colciencias, Bogotá, D.C., Colombia; Universidad \\ Nacional de Colombia, Bogotá, D.C., Colombia. \\ ${ }^{3}$ Departamento Administrativo de Salud del Vaupés, Mitú, Vaupés, Colombia. \\ ${ }^{4}$ Laboratorio de Micobacterias, Subdirección de Investigación y Desarrollo, Instituto Nacional de Salud, \\ Bogotá, D.C., Colombia.
}

Institución donde se realizó el trabajo: Departamento Administrativo de Salud del Vaupés, Mitú, e Instituto Nacional de Salud, Bogotá D.C., Colombia.

Se realizó una encuesta transversal en Mitú (Vaupés), un área de alta endemicidad para tuberculosis, con el objetivo de medir la proporción de sintomáticos respiratorios en la población, la prevalencia de casos de tuberculosis, de vacunación con BCG y la frecuencia de infección con Mycobacterium tuberculosis. Se incluyeron en el estudio 165 viviendas elegidas aleatoriamente por medio de un muestreo por conglomerados ( $n=20$ conglomerados) y se encuestaron 972 personas. La prevalencia de sintomáticos respiratorios fue de 3,6\% (IC95\% $2,6-4,9 \%$ ) mientras que la de vacunación con BCG fue de $94 \%$. Las personas vacunadas tenían una prevalencia menor de síntomas respiratorios (OR=0,37; IC95\% 0,15-0,95). La prevalencia de tuberculosis fue de $1,4 \%$ y se encontró que las personas con cicatriz de BCG tenían tres veces menos probabilidad de haber tenido tuberculosis (OR=3,3; IC95\% 1,0-14). Nuestros hallazgos sugieren que la proporción de sintomáticos respiratorios que el Programa Control de Tuberculosis tiene como meta de búsqueda (10\%) debe ser actualizada mediante una investigación operativa nacional. Asimismo, refuerzan la necesidad de lograr buenas coberturas de vacunación con BCG en poblaciones endémicas.

Palabras clave: tuberculosis, BCG, sintomático respiratorio, población indígena.

Respiratory syntomatic prevalence, infection and tuberculosis disease and associated factors: population-based study

A cross sectional survey on TB epidemiological characteristics was carried out in Mitú (Vaupes, Colombia) with the aim of measuring the prevalence of TB cases, the prevalence of TB suspected cases, the coverage with BCG vaccine and the prevalence of infection with Mycobacterium tuberculosis. One hundred and sixty five (165) households were included in the survey using a randomized cluster sampling design ( $n=20$ clusters) which yielded a sample size of 972 subjects. The prevalence of TB suspect cases was 3.6\% (C.I.95\% 2.6-4.9\%); coverage with BCG vaccine was $94 \%$. Vaccinated people had a lower chance of being a TB suspected case (OR=0.37 C.I.95\% 0.15-0.95). TB prevalence was $1.4 \%$. People vaccinated with BCG had a lower chance of having been a TB case (OR=3.3 C.I.95\% 1.0-14). These data recommend that $10 \%$ of people with respiratory symptoms be screened for in the National Control Program,and that the results be reviewed with surveys based at health centers. The data also reinforce the need for better vaccination coverages with BCG in high endemic areas.

Key words: tuberculosis, BCG, indigenous population, respiratory syntomatic patients. 
La tuberculosis continúa constituyendo un serio problema de salud en la Región de las Américas, donde cada año se notifican cerca de 250.000 enfermos y fallecen alrededor de 20.000 por esta causa (1). Según la Organización Mundial de la Salud, Colombia se ubica en el rango de 50 a 99 casos por 100.000 habitantes (2) aunque para el 2002 la incidencia notificada fue de 26,2 por 100.000 habitantes (3).

Históricamente, el departamento del Vaupés ha sido una de las áreas más endémicas para esta patología; presentó en el 2002 una tasa de incidencia de 70,4 por 100.000 habitantes. Algunas de las causas para esta elevada incidencia que hacen difícil la aplicación de las medidas de promoción y prevención en este departamento son: el $100 \%$ de la población indígena con necesidades básicas insatisfechas (NBI) y barreras culturales y geográficas para acceder a los servicios de salud. Otros factores de riesgo presentes son el hacinamiento, la reducida ventilación e iluminación de las viviendas y una deficiente nutrición (4).

El programa de control de tuberculosis tiene como parámetro que el $10 \%$ de los asistentes a consulta externa por primera vez por año, son sintomáticos respiratorios y deben ser sometidos a procedimientos de diagnóstico para tuberculosis (5); en Colombia, no se cuenta con esta información para la población general mientras que en otros países latinoamericanos, como Perú, se ha encontrado que la prevalencia de sintomáticos respiratorios en la población general es de $5 \%$ a nivel nacional (6).

En el presente estudio, realizado en marzo de 2001, el equipo interdisciplinario del Servicio Seccional de Salud del Vaupés y el Instituto Nacional de Salud se propuso medir la prevalencia de sintomáticos respiratorios y la cobertura de BCG en la población general de una localidad altamente endémica con el fin de mejorar el

\footnotetext{
Correspondencia:

Ingrid García, Avenida calle 26 N51-60, Bogotá, D.C., Colombia.

Teléfono: (1) 2207700 , ext. 150 y 111; fax: (1) 2200934.

igarcia@ins.gov.co o ingave@yahoo.com)

Recibido: 20/07/03; aceptado: 16/03/04
}

conocimiento que tenemos en el país sobre estos indicadores del programa de control. Igualmente, se evaluó la prevalencia de infección entre sintomáticos respiratorios y contactos de casos confirmados de tuberculosis.

\section{Materiales y metodos}

Tipo de estudio: se llevó a cabo una encuesta de corte transversal en la población de Mitú.

Tamaño de la muestra: se estimó que para encontrar una prevalencia de $10 \%$ con una precisión absoluta de $2 \%$ y un efecto de diseño de 2,0 se requerían alrededor de 800 personas. Al estimar que el promedio de habitantes por vivienda en Mitú debería estar entre 5,5 y 6 , se calculó que deberían visitarse, al menos, 150 viviendas. Finalmente, se decidió visitar 170 viviendas con el fin de cubrir un porcentaje de no respuesta estimado de $10 \%$.

Selección de la muestra: los participantes se seleccionaron con un muestreo por conglomerados monoetápico. Inicialmente, se seleccionaron 20 manzanas de 87 elegibles y, luego, se seleccionaron para la visita de campo 10 viviendas dentro de cada manzana. Las viviendas se eligieron en forma secuencial siguiendo el sentido de las manecillas del reloj.

Definiciones de caso: se definió como 'sintomático respiratorio' a toda persona que refería tos y expectoración por más de 15 días. Se definió como 'caso de tuberculosis' a toda persona que refería estar o haber estado en tratamiento para tuberculosis bajo la supervisión de un servicio de salud.

Instrumentos y procedimientos de campo: para realizar el estudio se diseñaron dos formatos para la recolección de datos, uno en forma de censo y otro en forma de encuesta estructurada.

En el censo domiciliario, se incluyeron las variables de persona, lugar y tiempo necesarias para ubicar geográficamente la vivienda, identificar a los individuos (edad, sexo, etnia) y procedencia, afiliación al sistema de seguridad social, identificación de sintomáticos respiratorios actuales, antecedentes de tuberculosis con diagnóstico y tratamiento y vacunación con BCG. 
Para verificar el estado vacunal, se revisó si cada miembro de la familia tenía o no la cicatriz vacunal de la BCG. Estas variables se analizaron, principalmente, para los sintomáticos respiratorios que era el grupo de interés de este estudio.

En cada vivienda seleccionada, se aplicaron los instrumentos elaborados (censo y encuesta); se entrevistaron las personas cabeza de familia o los adultos responsables que dieran información confiable.

La encuesta se aplicaba a las personas que cumplían con los siguientes requisitos: sintomáticos respiratorios, personas con antecedentes de tuberculosis y los contactos de los últimos. La encuesta contenía las variables necesarias para evaluar el tiempo de evolución de los síntomas, la tendencia que tiene la gente a consultar por estos síntomas, el sistema de salud al cual acude (tradicional, occidental), los procedimientos diagnósticos practicados y el registro de las pruebas de laboratorio tomadas para efectos del estudio con fecha de toma de muestra, prueba y resultado (baciloscopia seriada de esputo, cultivo y tuberculina). Se realizó una prueba piloto de aplicación del formulario con la cual se identificaron las preguntas más adecuadas para el estudio y la forma de hacerlas mejor para adaptarlas al lenguaje local.

A los sintomáticos respiratorios encontrados, se les practicaron pruebas diagnósticas de laboratorio: baciloscopia seriada de esputo, Ziehl Neelsen, cultivo de esputo por la técnica de Ogawa modificada por Kudoh (7) y prueba de tuberculina por multipunción monoprueba de Aventis Pasteur (8). A los sintomáticos respiratorios se les dieron las indicaciones pertinentes para la recolección de las muestras de esputo seriado que fueron recogidas por los encuestadores personalmente en las viviendas de los encuestados para evitar la pérdida de muestras durante los tres días consecutivos en que se hizo esta recolección; y la aplicación de la PPD a los mismos (figura 1). La prueba consistió en aplicar la PPD monoprueba de múltiple punción en la cara anterior del antebrazo izquierdo de acuerdo con las indicaciones de los fabricantes (9). La lectura se realizó a las 72 horas con la medición del diámetro

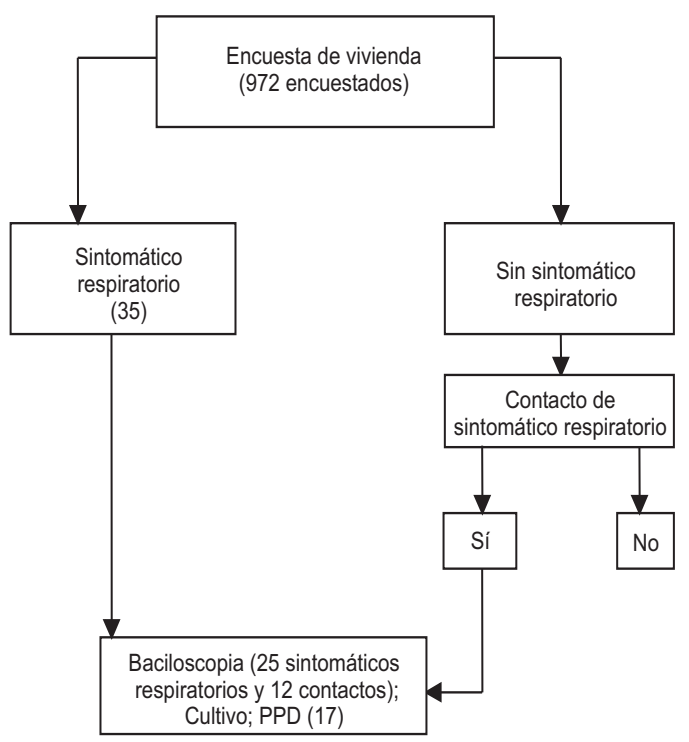

No se les logró hacer la totalidad de exámenes a todos los sintomáticos respiratorios.

Figura 1. Diagrama de flujo de los encuestados, Mitú, 2001.

de induración. EI PPD se interpretó de la siguiente manera: negativo: 0-1 $\mathrm{mm}$; dudoso: $2-4 \mathrm{~mm}$; positivo: 5 y más $\mathrm{mm}$. Con el fin de evitar sesgos de mala clasificación por la interpretación de la induración, se realizó una sesión de entrenamiento a los entrevistadores. En esta práctica, se les instruyó sobre cómo aplicar y leer la prueba usando algunos voluntarios seleccionados entre los encuestadores.

Validación de los resultados de tuberculina multipunción: debido a que, actualmente, el país no dispone de PPD RT23 estándar necesaria para la cuantificación de infección, se ha introducido en el mercado la prueba de multipunción Monotest para sustituirla. Dado que no existen reportes en la literatura de estudios nacionales de validación de este tipo de PPD contra el estándar de oro, se decidió evaluar la sensibilidad de esta nueva prueba para detectar infección comparándola con el estándar de oro PPD RT23 utilizando una muestra de individuos que tenían una respuesta conocida a la PPD RT-23 y que han mantenido esta respuesta en el tiempo en el rango de 0 a 21 $\mathrm{mm}$.

Manejo y análisis de datos: para manejar y procesar la información, se diseñó una base de 
datos utilizando el programa Epiinfo 6.04d. Se realizaron análisis descriptivos de las frecuencias de sintomáticos respiratorios, cicatriz de BCG y haber tenido tuberculosis. Luego, estas mismas características se analizaron por etnia, edad y sexo, afiliación al sistema de seguridad social. Se usó la razón de prevalencias (RP) y sus intervalos de confianza (IC 95\%) para medir la asociación entre variables dependientes e independientes. Se utilizó el programa Epiinfo 2002 para construir modelos multivariados (regresión logística) con el fin de controlar sesgos de confusión e identificar las variables independientes asociadas con ser sintomáticos respiratorios, estar vacunado y ser caso de tuberculosis.

\section{Resultados}

Características de la población: en el censo se incluyeron 165 viviendas y se entrevistaron 972 personas de los 16 barrios del casco urbano del municipio de Mitú. Los indígenas representaron el $51,4 \%$ de la muestra mientras que las mujeres eran el 50,8\%. La mediana de edad era de 19 años con un rango de 1 mes a 85 años. La proporción de hombres entre los indígenas fue del $46 \%$ y entre los no indígenas de $54 \%(p<0,01)$ aunque no hubo diferencias por edad entre los dos grupos. Entre la población no indígena, sólo 16,5\% era vinculado, mientras que, entre la población indígena, este porcentaje era mayor $(22,5 \%)$ $p<0,01$ (cuadro 1).

Validación de los resultados de tuberculina multipunción: los resultados de las lecturas de las pruebas de tuberculina Monotest comparada con el PPD RT 23, por métodos de regresión lineal mostraron un coeficiente de correlación de $r=0,84$ $r^{2}=0,7$ (IC 95\% 0,07-0,93).

Prevalencia de sintomáticos respiratorios: la prevalencia de sintomáticos respiratorios entre toda la población encuestada fue del 3,6\% (35/ 972) (IC 95\% 2,57-4,93) mientras que fue de 4,4\% (IC 95\% 2,9-6,7) para la población indígena. No se encontraron diferencias por sexo pero sí por edad. El $48,6 \%$ de los sintomáticos respiratorios se encontraron en el rango de edad de 15 a 44 años; el grupo con mayor prevalencia fue el de mayores de 60 años con $11,6 \%$ seguido por el grupo de 45 a 59 años con $5,45 \%$ (cuadro 2). El $37 \%$ (13) de los sintomáticos respiratorios consultaron a un médico, frente a $63 \%$ (22) que no consultaron.

La prevalencia de sintomáticos respiratorios entre las personas con cicatriz de BCG fue de $2,8 \%$, inferior a la de los no vacunados, $7,6 \%(\mathrm{RP}=0,37$; IC 95\% 0,15-0,95, $p=0,03$ ) (cuadro 2). Esta diferencia era mayor entre la población indígena donde la proporción de sintomáticos respiratorios era de $3,8 \%$ en población vacunada y de $9,8 \%$ en no vacunados (cuadro 3 ).

Se construyó un modelo de regresión logística en el cual se incluyo como variable dependiente ser sintomáticos respiratorios y como variables independientes la edad, la etnia y la presencia de cicatriz. Las variables que permanecieron asociadas estadísticamente fueron la edad y la presencia de cicatriz (OR=2,85; IC 95\% 1,03-7,8). A todos los sintomáticos respiratorios se les instruyó para que se practicaran un nuevo examen de baciloscopia y cultivo si continuaban con la sintomatología respiratoria. Igual recomendación fue hecha a los trabajadores de salud del programa con el fin de que buscaran a aquellos sintomáticos respiratorios que no consultaran nuevamente.

Prevalencia de población vacunada: el $92 \%$ de la población encuestada tenía cicatriz de BCG. Los niños menores de un año tenían una cobertura de $83 \%$, mientras que en los de 1 a 4 años aumentaba a $94 \%$. Luego, se mantenía alrededor

Cuadro 1. Distribución de la población encuestada de acuerdo con la afiliación al SGSSS, enero, 2001.

\begin{tabular}{lcccccc}
\hline Afiliación a Seguridad Social & \multicolumn{2}{c}{ Indígena } & \multicolumn{2}{c}{ No indígena } & \multicolumn{2}{c}{ Total } \\
\hline Contributivo & 197 & 39,4 & 220 & 46,6 & 417 & 42,9 \\
Subsidiado & 189 & 37,8 & 174 & 36,9 & 363 & 37,3 \\
Vinculado & 114 & 22,8 & 78 & 16,5 & 192 & 19,8 \\
Total & $\mathbf{5 0 0}$ & $\mathbf{5 1 , 4}$ & $\mathbf{4 7 2}$ & $\mathbf{4 8 , 6}$ & $\mathbf{9 7 2}$ & $\mathbf{1 0 0}$ \\
\hline
\end{tabular}


Cuadro 2. Distribución de variables de interés entre los sintomáticos respiratorios, enero, 2001.

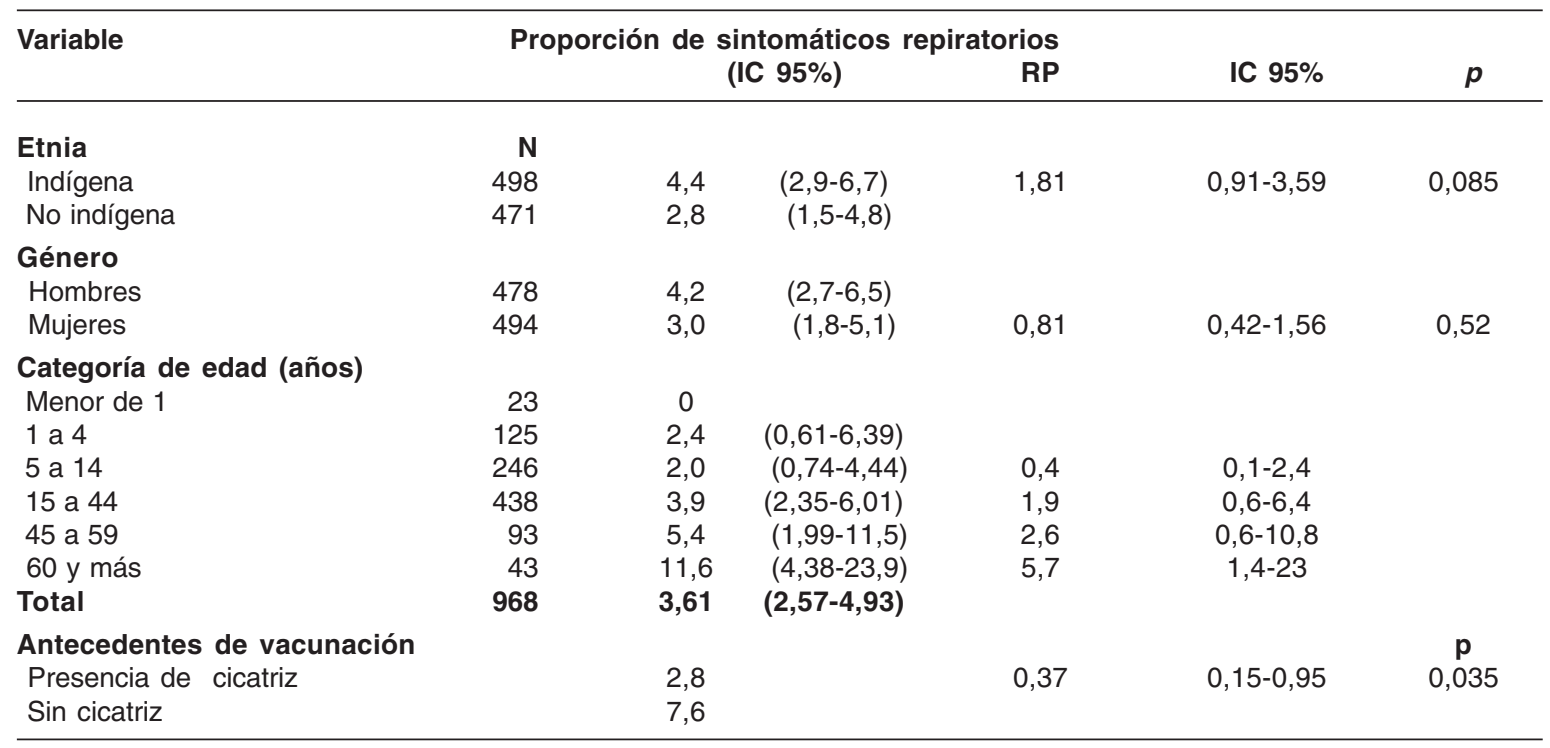

Cuadro 3. Distribución de las personas con antecedentes de vacunación con BCG por algunas variables de interés, enero, 2001.

\begin{tabular}{|c|c|c|c|c|c|}
\hline Variable & & $\begin{array}{l}\text { Proporción } \\
\text { con cicatriz }\end{array}$ & $\mathbf{R P}$ & IC 95\% & $\mathbf{p}$ \\
\hline \multicolumn{6}{|l|}{ Etnia } \\
\hline \multirow{2}{*}{\multicolumn{2}{|c|}{$\begin{array}{l}\text { Indígena }(n=440) \\
\text { No indígena }(n=405)\end{array}$}} & 90,7 & & & \\
\hline & & 93,8 & 0,97 & $0,93-1,01$ & 0,05 \\
\hline Categoría de edad (años) & $\mathbf{N}$ & $\begin{array}{l}\text { Proporción } \\
\text { de vacunados }\end{array}$ & & IC $95 \%$ & \\
\hline Menor de 1 & 23 & 82,6 & & $63,1-94,2$ & \\
\hline 1 a 4 & 117 & 94,0 & & $88,5-97,3$ & \\
\hline 5 a 14 & 217 & 92,6 & & $88,5-95,5$ & \\
\hline 15 a 44 & 373 & 92,2 & & $89,1-94,6$ & \\
\hline 45 a 59 & 77 & 92,2 & & $84,4-96,7$ & \\
\hline 60 y más & 38 & 89,5 & & $76,5-96,5$ & \\
\hline \multirow[t]{3}{*}{ Total } & 779 & 92,18 & & $90,2-93,8$ & \\
\hline & \multicolumn{2}{|c|}{ Sintomático respiratorio } & $\mathbf{R P}$ & IC 95\% & \\
\hline & unado & No vacunados & & & \\
\hline Indígenas & & 9,8 & 0,45 & $0,06-3,61$ & 0,45 \\
\hline No indígenas & & 4,0 & 0,39 & $0,13-1,11$ & 0,07 \\
\hline
\end{tabular}

de este nivel hasta los 60 años donde nuevamente la cobertura descendía (89\%) (cuadro 2).

Se construyó un modelo de regresión logística en el cual se incluyo como variable dependiente tener cicatriz y como variables independientes la edad y la etnia. La variable indígena permaneció asociada con la presencia de cicatriz aunque el valor de la $p$ de Wald no era significativo; sin embargo, la prueba de la razón de probabilidades sí lo fue (OR=1,56; IC 95\% 0,93-2,6, $p_{\text {wald }}=0,09$, $\left.p_{\mathrm{LRT}}=0,04\right)$.

Prevalencia de tuberculosis: la proporción de personas que estaba o estuvo en tratamiento para tuberculosis fue de 1,4\%; se encontró que los mayores de 60 años tenían una mayor prevalencia $(11,1 \%)$. El $85,7 \%$ correspondía a población 
indígena, $57,1 \%$ eran mujeres y $75 \%$ tenía cicatriz de vacunación $B C G$.

Entre los indígenas, la prevalencia de tuberculosis era 5 veces mayor que entre los no indígenas $(\mathrm{RP}=5,66 ;$ IC 95\% 1,27-25,17, p<0,001). También era mayor la prevalencia en población vinculada $(28,6 \%)$ y entre los sintomáticos respiratorios $(14,3 \%)$. Estos últimos tenían una probabilidad 14 veces más alta de estar o haber estado en tratamiento para tuberculosis que los no sintomáticos respiratorios (RP=14,79; IC 95\% $5,23-41,8, p<0,001)$. Por el contrario, la prevalencia de tuberculosis era $75 \%$ menor entre los que tenían cicatriz de BCG que entre los que no tenían ( $R P=0,25 ;$ IC 95\% 0,07-0,92, $p=0,02$ ).

Se construyó un modelo de regresión logística en el cual se incluyo como variable dependiente estar o haber estado en tratamiento para tuberculosis y como variables independientes la edad, la etnia y el tener cicatriz. Ser indígena y no tener cicatriz se mantuvieron asociados con un incremento en la probabilidad de haber tenido tuberculosis; las personas sin cicatriz tenían tres veces más riesgo de estar o haber estado en tratamiento para tuberculosis (OR=3,3; IC 95\% 1,0-14; $p=0,05)$, mientras los indígenas tenían 4 veces más riesgo de estar o haber estado en tratamiento para tuberculosis (OR=4,4; IC 95\% 1,0-20,3; $p=0,05$ ).

Prevalencia de infección: se le practicó prueba de tuberculina a 36 de las personas encuestadas; de ellos, el $58,3 \%$ eran sintomáticos respiratorios y el $40 \%$ restantes eran contactos de pacientes con tuberculosis. El 27,8 \% (10) de ellos tuvo un diámetro de induración mayor de $5 \mathrm{~mm}$ y fueron clasificados como 'positivo' de acuerdo con las instrucciones del PDD utilizado; un diámetro entre 2 y $4 \mathrm{~mm}$ en el $36,1 \%$, y entre 0 y 1 en el $36,1 \%$. Entre los positivos con un diámetro de 5 o más $\mathrm{mm}$, la proporción de indígenas fue de $70 \%$; el $30 \%$ tenía antecedentes de tuberculosis y el $60 \%$ eran sintomáticos respiratorios; la proporción de hombres fue del $70 \%$ y el $60 \%$ presentaba cicatriz.

De las personas con un PPD $<5 \mathrm{~mm}, 69,2 \%$ eran sintomáticos respiratorios, $84,6 \%$ eran indígenas, ninguna tenía antecedente de tuberculosis y $90.9 \%$ presentaba cicatriz.
Se realizaron 105 baciloscopias a 35 personas; de ellas, $12(32,5 \%)$ eran contactos de algún caso de tuberculosis; las muestras fueron en su totalidad negativas para bacilos ácido alcohol resistentes; igualmente, se realizaron cultivos de estas muestras que fueron negativos.

\section{Discusión}

En Colombia, no encontramos estudios publicados en los que se haya determinado la proporción de sintomáticos respiratorios en la población general; por lo tanto, ésta podría ser la primera aproximación en demostrar que la prevalencia de sintomáticos respiratorios en la población general, aun de una región de alta endemicidad, no es tan alta como podría creerse, ya que fue de $3,6 \%$ en la población total y de $4,4 \%$ entre la población indígena. Nuestros resultados son comparables con los realizados a gran escala en otros países latinoamericanos, como Perú, que reporta un $5 \%$ de sintomáticos respiratorios entre la población general a nivel nacional (10); igualmente comparables con estudios realizados en países no latinoamericanos que han encontrado esta cifra cercana al $5 \%$ como Uganda (4,1\%), Burkina-Faso (3,5\%) e Indonesia $(3,7 \%)(10)$.

A partir de los estudios de K. Styblo (11), los programas de control de tuberculosis optaron, principalmente, por la búsqueda pasiva de casos en la consulta general, para lo cual algunos países como Colombia investigaron la frecuencia de sintomáticos respiratorios entre los consultantes de primera vez/año; se encontró un $10 \%$, lo cual constituye actualmente la meta operativa en la norma oficial del Ministerio de la Protección Social. Debido a que fueron estudios realizados hace mucho tiempo, es de vital importancia actualizarlos mediante una investigación operativa nacional por ser un punto de alta importancia en el control de la tuberculosis ya que permitiría ayudar a estimar de manera más real la magnitud de la búsqueda y el diagnóstico de pacientes que se requiere realizar.

Otro punto de importancia en el presente estudio es haber podido medir la proporción de sintomáticos respiratorios que consultan a los 
servicios de salud, lo que también puede apoyar al programa en el diseño de mejores estrategias de promoción y prevención (12).

Sorprendentemente, el $63 \%$ de los sintomáticos respiratorios no había consultado a ninguna institución, lo que sugiere que el impacto de un sistema de búsqueda y control basado sólo en los servicios asistenciales podría ser más bajo de lo pensado. Por ello, se hace necesario que, en los sitios de alta endemicidad, los prestadores de servicios inviertan más en actividades de promoción y prevención basadas en la comunidad que conduzcan a la detección de más sintomáticos respiratorios. Se debe tener especial cuidado en la vigilancia que se realice en la población de 60 y más años ya que son el grupo más vulnerable, además de los menores de un año. También, se debe priorizar la búsqueda activa en indígenas, quienes tenían casi el doble de sintomáticos respiratorios que los no indígenas. Otros estudios en nuestro país han demostrado que los indígenas tienen mucha más probabilidad de enfermar de tuberculosis que las poblaciones no indígenas a su alrededor dadas sus condiciones nutricionales y de hacinamiento $(9,13)$.

Otro hallazgo importante es la asociación protectora encontrada entre estar vacunado con BCG y tener o haber tenido tuberculosis. En nuestro estudio, la protección es de una magnitud muy importante $(\sim 70 \%)$ aunque la precisión es baja por el escaso número de casos, este efecto protector de la vacuna persiste aún después de controlar por otros factores y es más alto que lo encontrado en otros estudios (14). Este hallazgo debe ser interpretado con precaución dado que es posible que parte de esta magnitud sea explicada por el diseño del estudio. Éste fue un estudio de corte transversal y, por ello, los factores asociados con la mortalidad tienden a quedar sobrerepresentados o subrepresentados, dependiendo de si son protectores o promotores de la misma. En este caso, uno pensaría que los casos de tuberculosis con antecedente negativo de BCG tenderían a morir más que los no vacunados por lo que nuestro estudio tendería a sobreestimar la asociación entre BCG y tuberculosis. Sin embargo, aun asumiendo que la diferencia en la mortalidad sea del doble - es decir, que los no vacunados con tuberculosis tengan una letalidad dos veces mayor que los vacunados $y$, por tanto, una probabilidad disminuida de ser incluidos en un estudio transversal - aun la vacuna disminuiría la posibilidad de haber tenido tuberculosis en un $30 \%$ o $40 \%$ lo cual es importante desde el punto de vista de salud pública. Por ello, la vacunación con BCG debe ser fortalecida y realizada de manera intensificada en estas poblaciones endémicas $(12,15)$.

La prevalencia de infección tuberculosa medida por el PPD entre personas sintomáticas y contactos fue de $27,8 \%$; es la mayor prevalencia entre indígenas, en el grupo de mayores de 60 años y el género masculino con $70 \%$. Esta prevalencia es similar a la encontrada en estudios realizados en poblaciones de alto riesgo de infección tuberculosa, como los realizados en inmigrantes en Ceuta, España, (32,6\%) (16) y entre estudiantes del área de la salud con PPD superior a $15 \mathrm{~mm}$ en un $30 \%$ (17).

Los pacientes que tuvieron una reacción negativa al PPD, contrario a lo que se pensaría, tenían antecedentes de vacunación en $90,9 \%$, lo que validaría el uso de esta prueba para estudios epidemiológicos aun en poblaciones de alta circulación de M. tuberculosis y de alta cobertura con BCG, siempre y cuando se tenga la certeza de una buena calidad en el proceso de vacunación y se descarte la ausencia del fenómeno de refuerzo y la anergia (18).

En relación con la prueba de tuberculina usada en el campo, es importante anotar que hay algunas fallas en su presentación que pueden alterar el valor de los resultados cuando se usa en manos inexpertas. En primer lugar, el inserto de la prueba no trae en forma clara las unidades de tuberculina que contiene cada dosis; tampoco especifica la cantidad contenida en cada uno de los dispositivos, ni se especifica su equivalencia biológica con respecto a los patrones internacionales; además, el producto no especifica la temperatura de conservación. También, se debe resaltar que aunque en la prueba de validación hubo diferencias entre los resultados de la prueba multipunción y los que se tenían de un grupo de personas en el Laboratorio de Micobacterias del 
Instituto Nacional de Salud, la concordancia entre las dos pruebas fue alta $(r=0,84)$ lo que sugiere que los resultados de prevalencia de infección medidos por la prueba de multipunción son un estimador muy cercano de la verdadera prevalencia. De todas maneras, se debe impulsar el uso del PPD RT-23 recomendado por la OPS/ OMS dentro del programa de control de la tuberculosis de Colombia del cual, por razones desconocidas, ha sido retirado. Se podría llevar a cabo un estudio más amplio de comparación entre las diferentes pruebas de tuberculina que se encuentran comercialmente disponibles en el país con el fin de unificar los criterios de positividad e identificar el punto de corte de la tuberculina Monotest en la población colombiana, para lo cual la población podría ser niños en edad escolar tuberculino negativo y sin cicatriz de BCG.

\section{Agradecimientos}

A la comunidad del casco urbano del municipio de Mitú que amablemente participó en el estudio y a los funcionarios del Servicio Seccional de Salud del Vaupés que participaron en el cursotaller de capacitación en VSP en el departamento del Vaupés, organizado por la Subdirección de Epidemiología y LNR del Instituto Nacional de Salud, al Laboratorio del Hospital San Antonio de Mitú y el LSP del Vaupés. A Clara Eugenia González, por su colaboración en la validación de los resultados de la tuberculina multipunción.

\section{Referencias}

1. World Health Organization. Global tuberculosis control: surveillance, planning, financing. WHO/CDS/ TB/2003.316. Geneva, Switzerland: World Health Organization;2003.

2. World Health Organization. Global tuberculosis control: surveillance, planning, financing. WHO/CDS/ TB/2002.295. Geneva, Switzerland: World Health Organization;2002.

3. Ministerio de Salud. Estadísticas del Programa de Control de Tuberculosis. Informes anuales. Archivos del Ministerio de Salud. Bogotá, Colombia 1970-2002.

4. Guevara J. En busca de un modelo de salud para los pueblos indígenas. La experiencia del Vaupés. Servicio Seccional de Salud del Vaupés. Bogotá: Minsalud; 1998.

5. Arciniegas A, Barrera MT, Guerrero E. Prevalence of tuberculosis among patients with respiratory symptoms who consult for the $1^{\text {st }}$ time the health establishments of Risaralda, Colombia. Bol Oficina Sanit Panam 1979;87:512-24.

6. Programa Nacional de Control de Tuberculosis, Perú. Tuberculosis en el Perú. Informe 1995. Lima, Perú: Talleres de Gráfica Edias; 1996.

7. Garzón MC, Naranjo ON, Sierra CR, Llerena C, Orjuela DL. Bacteriología del Mycobacterium tuberculosis y de micobacterias no tuberculosas. Manual de procedimientos. Bogotá, Colombia: Instituto Nacional de Salud; 2001.

8. Aventis Pasteur. Instrucciones para el uso de la tuberculina multifunción. Disponible en: http:// www.aventispasteur.com/usa/product/pdffiles/!LE6315.pdf

9. Henao G, de la Hoz F, León Cl, Ribón W, Guerrero MI. Epidemiología clásica y molecular de la tuberculosis en el Guaviare 1997-1998. Inf Quinc Epidemiol Nac 1999;4:85-91.

10. WHO. Control de la tuberculosis. Situación actual en documentos básicos. Control de tuberculosis. Washington, D.C.: Organización Panamericana de la salud; 1988 .

11. Borgdorff MW, Floyd K, Broekmans JF. Interventions to reduce tuberculosis mortality and transmission in low and middle income counties. Bull WHO 2002;80:217-27.

12. Rothman K. Epidemiology. An introduction. New York: Oxford University Press; 2002.

13. León CI, Sierra CR, Naranjo N, Garzón MC, Guerrero MI. Characteristics of the Colombian tuberculosis population (1999-2000). Abstracts CD. 6-6. 33th IUATLD World Conference on Lung Health. Stop TB, Fight Poverty, Symposium Satelite. 2002. Montreal

14. Arbeláez MP. La protección de la vacuna BCG contra la tuberculosis. Médicas UIS 1998;12:173-80.

15. Checkoway H, Pearce N, Brown CD. Research methods in occupational epidemiology. New York: Oxford University Press; 1989.

16. Fernández $M$, Díaz $P$, Sánchez J, Pérez A, Andrade A. Prevalencia de infección tuberculosa en la población de inmigrantes en Ceuta, España. Rev Esp Salud Pública 2001;75:551-8.

17. Arbeláez MP, Ocampo M, Montoya J, Jaramillo L, Giraldo P, Maldonado A, Cano E, Mejia O, García L. Evaluación de la respuestas a la tuberculina en estudiantes del área de salud. Rev Panam Salud Pública 2000;8:272-9.

18. Menzles R, Vissandjee B, Rocher I, St Germain Y. The booster effect in two-step tuberculin skin testing among young adults in Montreal. Ann Intern Med 1994;120:190-8. 